\title{
Experimental Research on Hydrophilic Property of Stainless Steel Bionic Non-smoothed Surface
}

\author{
Yan $\mathrm{Li}^{1, \mathrm{a}}$ *and Guangrui Shang ${ }^{1, \mathrm{~b}}$ \\ ${ }^{1}$ School of Mechanical Engineering at Jilin Engineering Normal University, Changchun Jilin 130052 , \\ China \\ aliyan0496@126.com, bshgr888@163.com
}

Keywords: Bionics; Crown height; Interval; Contact angle; Stainless steel

\begin{abstract}
The laser precision machining was performed on the surface of the formed spherical non-smooth lattice, leads to an increase in material surface hydrophilic tendency. The influence of ball crown height and interval on wettability has been studied. The results show that the ball crown lattices have been increased the apparent contact area between the water droplet and the surface leading to the decreasing of apparent contact angle up to $28.5 \%$ and the surface hydrophilicity is ameliorated.
\end{abstract}

\section{Introduction}

The factors that affect the wettability of the material were the interface tension of the material and the appearance of the material surface [1-9]. the field of mechanical engineering and medical engineering commonly used in the field of stainless steel as the research object, using laser precision processing technology constructed spherical cap shape non-smooth bionic surface, smooth spherical surface shape on the surface wettability of the material effects of micron level was investigated, which improved the hydrophilicity of the stainless steel (1Cr18Ni9Ti), provides a new idea for the improvement of the surface wettability of the material.

\section{Materials and Equipment}

The stainless steel cylindrical sample was $18 \mathrm{~mm}$ in diameter and the height was $10 \mathrm{~mm}$. After cutting and polishing, Surface roughness was less than 0.8 microns, and the sample was stored in the alcohol container. Using CNC laser engraving machine adjusted the strength of the current, fill spacing, step length and frequency to control the non-smooth spherical surface diameter, spacing, column spacing and the height. The surface topography analysis instrument was used for the analysis. Drops Method Sessile was used to complete the contact angle measurement, the instrument measurement angle range was 0-180 degrees, the measurement error was 0.1 degrees. The data was measured at 4 points, and the mean value was measured.

\section{Methods}

In order to study the non-smooth spherical lattice spherical diameter, spaced row interval, column spacing, and the altitude effect on the wettability. So we can by changing the current intensity, filling spacing, frequency and step to achieve. The specific range of choice for the current intensity of $130 \sim 280$, the filling spacing of $0.15 \sim 0.5 \mathrm{~mm}$, frequency $5 \sim 10 \mathrm{~Hz}$, the step size was $0.13 \sim 0.25$ $\mathrm{mm}$. Study on the influence of the surface wetting property of materials by mixed orthogonal test $L_{32}\left(8 \times 4 \times 2^{2}\right)$. The specific test was divided into 4 groups, a total of 32 times.

\section{Results}

Before the experiment, the intrinsic contact angle of the surface of the smooth stainless steel was first determined $\theta_{e}=84^{\circ}$. From the test results, it can be known that the wetting performance of the material surface has been significantly improved. Actual contact angle decreased by 23.93degrees, 
and the ratio was $28.8 \%$.

\section{Analysis and Discussion}

Test results of statistical description were shown in Table 1. The regression equation was as follows: Eq.1. The standard regression coefficient of the equation shows that the factors which influence the actual contact angle were $\mathrm{x}_{1}, \mathrm{x}_{4}, \mathrm{x}_{3}, \mathrm{x}_{2}$. Filling spacing $\left(\mathrm{x}_{1}\right)$ was the most significant influence on the actual contact angle.

$$
y=56.9020+49.7562 x_{1}-0.0376 x_{2}+0.1960 x_{3}+9.6893 x_{4}
$$

Table 1 Statistical analysis of experimental results

\begin{tabular}{|c|c|c|c|c|c|}
\hline Project & $\begin{array}{c}\text { Fill spacing } \\
{[\mathrm{mm}]} \\
x_{1}\end{array}$ & $\begin{array}{c}\text { Electric } \\
\text { current } \\
{[\mathrm{A}]} \\
x_{2}\end{array}$ & $\begin{array}{c}\text { Frequency } \\
{[\mathrm{Hz}]} \\
x_{3}\end{array}$ & $\begin{array}{c}\text { Step } \\
{[\mathrm{mm}]} \\
x_{4}\end{array}$ & $\begin{array}{c}\text { Actual contact } \\
\text { angle }\left[{ }^{\circ}\right]\end{array}$ \\
\hline Sum & 10.4 & 6560.00 & 240.00 & 6.08 & 2197.47 \\
\hline Average & 0.325 & 205.00 & 7.50 & 0.19 & 68.67 \\
\hline Max & 0.50 & 280.00 & 10.00 & 0.25 & 83.99 \\
\hline Min & 0.15 & 130.00 & 5.00 & 0.13 & 60.07 \\
\hline Covariance & 0.0131 & 3125.00 & 6.25 & 0.0036 & 47.01 \\
\hline Number & 32 & 32 & 32 & 32 & 32 \\
\hline
\end{tabular}

Effect of Element Parameters on the Hydrophilic Property. As shown in Fig. 1, the sample surface was refined with micron level spherical lattice near regular arrangement. The relationship between the lattice parameters and the actual contact angle was shown in Table 1. In this experiment, we studied the water droplets in the nearly regular non-smooth spherical cap surface wetting behavior. The results show that the change of laser processing parameters directly affects the surface morphology of the material, namely, the change of roughness factor ( $r$ ) or the pore fraction $\left(\Phi_{s}\right)$.The experimental results was shown that the wettability of stainless steel surface can be improved by changing the surface morphology. The actual contact angle was decreased by 23.93 degrees, and the decrease was $28.5 \%$ compared with the smooth plane.

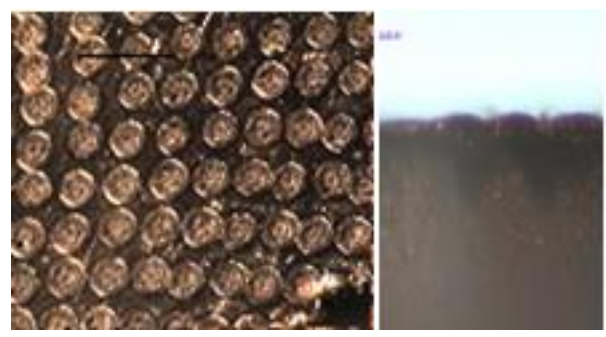

Figure 1. Morphology of surface (Processed), bar equals to 500 $\mu \mathrm{m}$

Effects of Crown Height on the Actual Contact Angle. Fig. 2 was the current processing under different cap height change on the actual contact angle curve. Curves was shown that regardless of how to adjust the current processing, the actual contact angle with the cap height increase showed a downward trend, but when the height of the spherical crown more than 40 microns, there tended to be gentle.

The Relation Ship between the Line Spacing and the Actual Contact Angle. Fig. 3 were line spacing to the actual contact angle the relation curves and corresponding surface morphology, with increase of the spacing, actual contact angle increasing; however, spacing in below, actual contact angle changes little. 


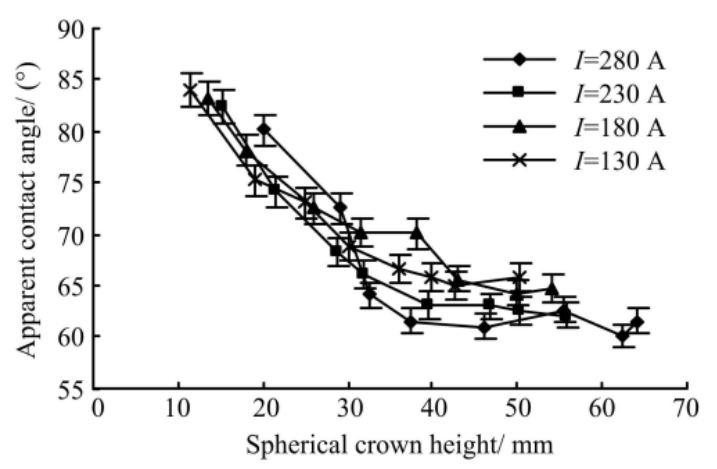

Figure 2. Curve of apparent contact angle by spherical crown height

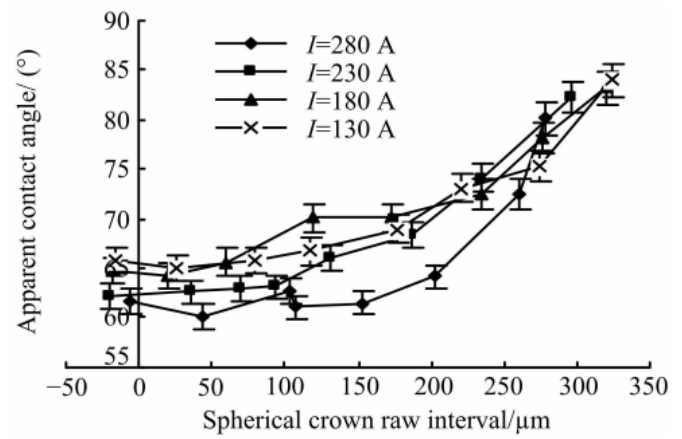

Figure 3. Influence of interval on apparent contact angle

The Change Trend of the Actual Contact Angle. According to the test results, unit of crown diameter and column spacing changes to the actual contact angle does not have a significant impact. Therefore, in the analysis can temporarily does not consider the unit sphere diameter and gap on the actual contact angle effect. From the Fig. 2 and Fig. 3, we can see that the actual contact angle in the process of gradually decreasing, there was a trend of flat. In this experiment, on the actual contact angle have significant effects of spherical crown height increase rate showed a decreasing trend, leading to slow down the water droplet and the surface of the real contact area rate of increase slowed down, the actual contact angle decreased.

Comparison with Wenzel [10] Model. In this test, the surface material after laser precision processing of the crown. Set spacing for L, column spacing was B, the spherical diameter spherical crown was D, and the crown height was $\mathrm{H}$, then their formula for Eq.2.

$$
r=\frac{\pi\left(H^{2}+0.25 D^{2}\right)+L B+B D+L D+4 \times\left(\frac{1}{4} D^{2}-\frac{1}{4} \times \frac{1}{4} \pi D^{2}\right)}{(B+D)(L+D)}==\frac{\pi\left(H^{2}+0.25 D^{2}\right)+L B+B D+L D+0.215 D^{2}}{(B+D)(L+D)}
$$

In this experiment, the relationship between the effect of the roughness factor (r) on the actual antenna angle and the calculated value was shown in Fig. 4. As can be seen from Fig. 4, there was a big difference between the theoretical value and the observed value. After calculation the actual contact angle was 61.55 degrees, Roughness factor (r) was approximately equal to 4.524, corresponding to the crown height was greater than or equal to 650 microns. In this experiment, it was not recorded. The statistical relationship between the observed value of the actual contact angle and the roughness factor was Eq.3.

$$
\theta^{*}=-25228 r^{4}+83277 r^{3}-91613 r^{2}+33646 r
$$

The relationship $\cos \theta^{*} / \cos \theta \sim f(r)$ curve was shown in Fig. 5. Its statistical relationship was Eq.4. After finishing was Eq.5.

$$
\cos \theta^{*} / \cos \theta=4025.2 r^{4}-13273 r^{3}+14575 r^{2}-5325.4 r
$$




$$
\cos \theta^{*}=\left(4025.2 r^{4}-13273 r^{3}+14575 r^{2}-5325.4 r\right) \cos \theta
$$

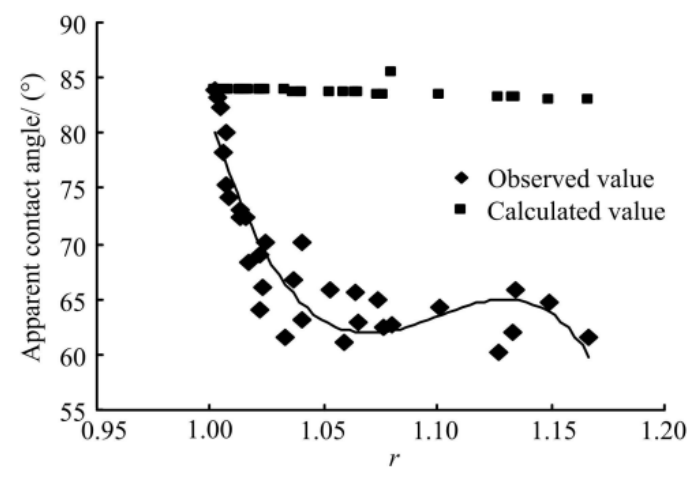

Figure 4. Curve of apparent contact angle by Roughness factor $r$ : Observed and Calculated Value

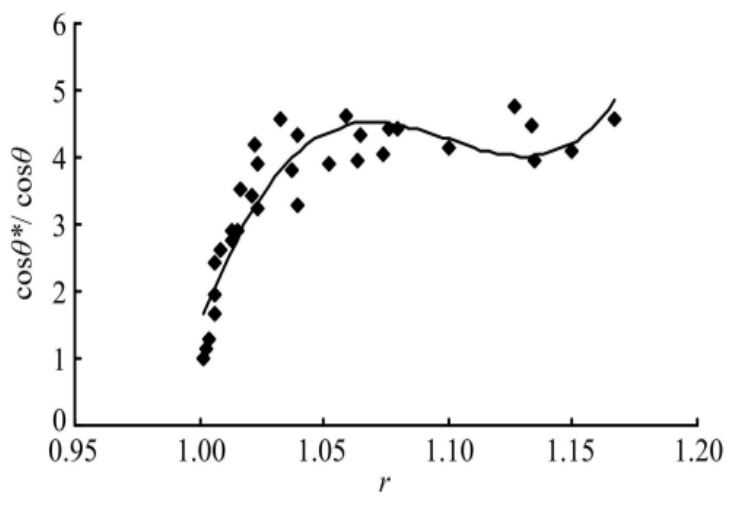

Figure 5. Curve of $\cos \theta^{*} / \cos \theta \sim f(r)$

Comparison with Cassis-Baxter [11] Model. Cassis-Baxter equation as follow: Eq. 6.

$$
\cos \theta_{c}=-1+\Phi_{s}\left(1+\cos \theta_{Y}\right)
$$

Note: $\Phi_{s}=\frac{\pi\left(H^{2}+0.25 D^{2}\right)}{(L+D)(B+D)}$

The relationship between the actual contact angle and the area fraction $\left(\Phi_{s}\right)$ was shown in Fig. 6 . In the curve shown in Fig. 5, the relationship between the area fraction and the actual contact angle was statistically significant. The relationship between the cosine value and the area fraction was shown in Fig. 7.

$$
\theta^{*}=-277.43 \Phi_{s}^{4}-73.239 \Phi_{s}^{3}+488.07 \Phi_{s}^{2}-288.95 \Phi_{s}+112.47
$$

Note: $\theta^{*}=\frac{\cos \theta^{*}-1}{\cos \theta-1}$. The regression equation was as follows: Eq.9. After finishing was Eq.10.

$$
\begin{aligned}
& \frac{\cos \theta^{*}-1}{\cos \theta-1}=-2.3289 \Phi_{s}^{4}-5.8178 \Phi_{s}^{3}+11.527 \Phi_{s}^{2}+5.9122 f \Phi_{s}+1.566 \\
& \cos \theta^{*}=\left(-2.3289 \Phi_{s}{ }^{4}-5.8178 \Phi_{s}^{3}+11.527 \Phi_{s}{ }^{2}+5.9122 \Phi_{s}+1.566\right)(\cos \theta-1)+1
\end{aligned}
$$

Similarly, the observed values were still not consistent with the Cassis model.

Through the above analysis, we can know that under the conditions of this experiment, that have been compared with the Wenzel model and the Cassis model. There were two reasons for this phenomenon: one was because that the spherical cell body diameter and row spacing or diameter and column spacing ratio was too large. Another reason was that the height and the diameter of spherical element ratio were too small.

The above reasons lead to the Wenzel model in the roughness factor was too small and Cassie model in the area fraction was too large, so there was a large deviation between the theoretical value and the observed value.

The above reasons lead to the value of the Wenzel model was too small and the value of the Cassie model was too large, then there was a large deviation between the theoretical value and the observed value. 


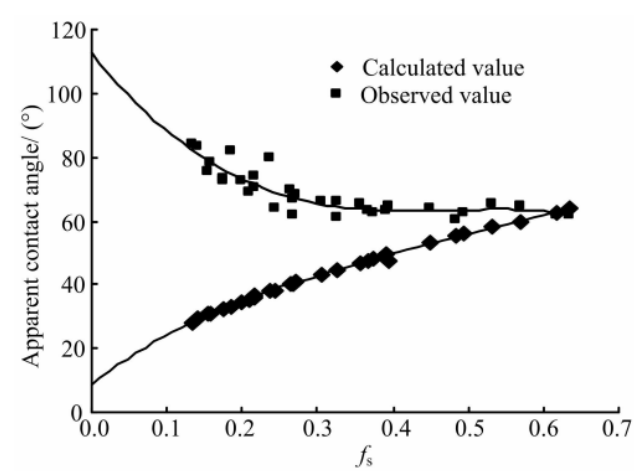

Figure 6. Curve of apparent contact angle by $\Phi_{s}$ : Observed and Calculated Value

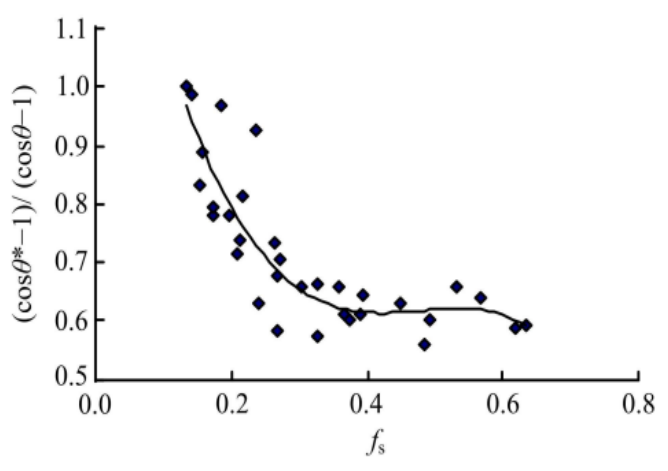

Figure 7. Curve of $\frac{\cos \theta^{*}-1}{\cos \theta-1} \sim f\left(\Phi_{S}\right)$

\section{Conclusion}

Non-smooth stainless steel bionics surface has been fabricated by laser precision processing and hydrophilic capability has been increased $28.8 \%$. The regression equation was Eq.1. Among the factors that influence factors such as the spacing, the current, the frequency and the step size, the effect of the spacing on the actual contact angle was the most significant.

When the rough factor ( $r$ ) was used as the parameter of the study, the formula was as Eq.3. When the area fraction $\left(\Phi_{s}\right)$ is used as the research parameter, the formula was as Eq.8

\section{References}

[1] K. Teshima, H. Sugimura, Y. Inoue, O. Takai, A. Takano, Wettablity of poly (ethylene terephthalate) substrates modified by a two-step plasma process: ultra water repellent surface fabrication, Chem. Vapor Depos., 2004, 10(6): 295-297.

[2] A. Nakajima. Design of a transparent hydrophobic coating. J. Ceram. Soc. Jpn, 2004, 112: 533-540.

[3] R. Furstner, W. Barthlott, C. Neinhuis, P. Walzel. Wetting and self-cleaning properties of artificial super-hydrophobic surfaces. Langniuir, 2005, 21(3):956-961.

[4] A.R. Parker, C.R. Lawrence. Water capture a desert beetle. Nature, 2001, 414: 3-34.

[5] J.P. Rothstein. Direct velocity measurements of the flow past drag-reducing ultrahydrophobic surfaces. Phys. Fluids, 2005, 17(10): 103606-103615.

[6] Abdelsalam, M. E.; Bartlett, P. N.; Kelf, T.; Baumberg, J. Wetting of Regularly Structured Gold Surfaces. Langmuir; 2005; 21(5);:1753-1757.

[7] R.Mohammadi, J. Wassink, A. Amirfazli, Effect of surfactants on wetting of super-hydrophobic surfaces,Langmuir,2004,20:9657-9662.

[8] M. Niemitz, G. Koßmehl, Wettability of poly (2, 2'-bithieny-5,5'-diyl) layers, Angew. Mackromol. Chem, 1991, 185: 147-154.

[9] S. Veeramasuneni, J. Drelich, J.D. Miller, G. Yamauchi, Hydrophobicity of ion-plated coatings, Prog. Org. Coat., 1997, 31: 265-270.

[10]Wenzel, R.N. Industrial and Engeneering Chemistry, 1936, 28, 988-994.

[11]Cassie, A.B.D.; Baxter, S. Transactions of the Faraday Society, 1944, 40,546-551. 